\title{
Desafios para o Planejamento e Implantação da Gamificação no Contexto Educacional
}

\author{
ARMANDO MACIEL TODA - armando.toda@gmail.com - Universidade de São Paulo (USP) \\ ALAN PEDRO DA SILVA - alan.pedro.da.silva@gmail.com - Universidade de São Paulo (USP) \\ SEIJI ISOTANI - sisotani@icmc.usp.br - Universidade de São Paulo (USP)
}

\begin{abstract}
Resumo:
A gamificação vem sendo difundida no contexto educacional como uma forma de aprimorar o engajamento e a motivação de alunos. Vários estudos mostram os resultados positivos de sua aplicação, o que faz com que as pesquisas nessa área estejam ganhando espaço na ciência. No entanto, para alcançar estes resultados, é necessário realizar um bom planejamento do contexto que está sendo sendo submetido para gamificação. Até o momento não há estudos que reportem problemas que podem ocorrer em contextos educacionais durante a fase de planejamento, a fim de evitá-los. Por conta disto, este trabalho propõe uma análise de estudos secundários (revisões sistemáticas e da literatura), visando a identificação destes problemas referentes às fases de planejamento e implantação da gamificação. No total, foram analisados onze trabalhos e identificados seis problemas relacionados ao design da gamificação. A partir da identificação desses obstáculos, este trabalho fornece uma base para que futuras abordagens de gamificação possam evitá-los durante o planejamento e implantação.
\end{abstract}

Palavras-chaves: gamificação, revisão sistemática, design

\begin{abstract}
:
Gamification has been widely widespread in educational context as a way to improve students' engagement and motivation. Many studies demonstrate the positive effects of its applications, which increases its popularity among scientific area. However, in order to achieve these positive results, it is necessary to perform a good planning of what contexts are intended to gamify. Until now, there is a lack of studies that report the problems that can occur in educational contexts during planning and implanting phase of gamification, in order to avoid them. Therefore, this work propose an analysis of secondary studies (systematic reviews and literature reviews) aiming at identifying the problems that can occur in planning and implanting phases of gamification. In total, six problems were mapped, related to gamification design, as: lack of empirical evidence, lack of definitions, lack of specific methods, customization, undesired behavior, lack of technological support. Furthermore, through the identification of these obstacles, this work provides a base so that future gamification approaches may avoid them during the planning and implementation phases.
\end{abstract}

Keywords: gamification, systematic review, design 


\section{Introdução}

A gamificação vem sendo usada amplamente em contextos educacionais nos últimos anos (Borges et al., 2014). Definida como a utilização de elementos de jogos fora do contexto de jogos, a gamificação pode ser utilizada para aprimorar a motivação e o engajamento, assim como servir de apoio para processos de ensino e treinamento (Kapp, 2012; Deterding et al., 2011). No contexto educacional, a gamificação está presente tanto em ambientes virtuais de aprendizagem (Toda et al., 2014; Klock et al., 2015; Challco et al., 2016) quanto na própria sala de aula (Toda et al., 2016). Diversos trabalhos discutem os benefícios relacionados ao uso da gamificação em ambientes educacionais, dentre eles os mais citados são a melhoria no engajamento, no processo de aprendizagem ou maestria de habilidades e, também, mudanças positivas de comportamento (Borges et al., 2014). Apesar dos diversos resultados positivos apresentados na literatura, estes só podem ser alcançados a partir de um bom planejamento (Dicheva et al., 2015; Zichermann \& Cunningham, 2011; Andrade et al., 2016).

O planejamento e implantação da gamificação é uma importante área de pesquisa e vem recebendo maior atenção nos últimos anos (Nacke \& Deterding, 2017). Apesar de sua importância, muitos estudos sobre gamificação não utilizam métodos adequados de planejamento de gamificação que levam em consideração tanto as teorias de design de jogos quanto o contexto onde os elementos de jogos serão utilizados.

Para lidar com esse problema, diversos pesquisadores vêm propondo abordagens sistemáticas como uma forma de auxiliar o design da gamificação. Apesar disso, até o momento existe uma carência de pesquisas que fazem análises aprofundadas sobre os problemas relacionados ao planejamento e implantação da gamificação (Nacke \& Deterding, 2017; Kim \& Werbach, 2016).

Baseando-se no exposto, este artigo apresenta a seguinte pergunta de pesquisa: "Quais as principais dificuldades relacionadas ao planejamento e implantação da gamificação em contextos educacionais?". Para responder esta pergunta de pesquisa, foi realizada uma revisão sistemática da literatura com o intuito de identificar problemas relacionados ao planejamento e implantação da gamificação. Foram analisados onze estudos secundários (revisões de literatura e sistemáticas) e identificados seis problemas.

Para apresentar a proposta, este trabalho está estruturado da seguinte forma: Seção 2 apresenta um conjunto de obstáculos e efeitos colaterais relacionados a gamificação; Seção 3 apresenta o protocolo da revisão; Seção 4 apresenta os resultados encontrados e uma breve discussão; Seção 5 apresenta as conclusões e trabalhos futuros.

\section{Efeitos Colaterais da Gamificação na Educação}

Problemas encontrados no planejamento e/ou implantação da gamificação são escassos na literatura, grande parte dos trabalhos focam nos problemas pós-implantação, quando mensuram o comportamento intencionado da forma correta. Kim \& Werbach (2016) 
mapearam um conjunto de problemas éticos relacionados a gamificação de forma geral, como:

- Exploração: onde os designers aplicam conceitos de gamificação de forma superficial e não alcançando os benefícios intencionados;

- Manipulação: onde a gamificação implementada é utilizada para manipular a mudança de comportamento do usuário;

- Danos físicos: onde os elementos de jogos afetam a percepção do usuário podendo causar acidentes;

- Danos psicológicos: onde estes mesmos elementos podem gerar ambientes não saudáveis para os envolvidos, como o mal planejamento de um placar;

- Influência negativa nos traços de personalidade do usuário: onde os elementos aplicados podem encorajar os jogadores a serem indiferentes quanto a valores humanos fundamentais.

Outro estudo que reporta problemas relacionados a gamificação é o de Thiebes, Lins e Basten (2014). Neste estudo, os autores focam em analisar quatro riscos que a gamificação de sistemas de informação deve considerar quando utilizar os elementos de jogos, sendo:

- Qualidade da tarefa: onde a gamificação influencia na qualidade das ações que devem ser desempenhadas, de modo que os elementos de jogos atrapalham ou desviam a atenção do jogador;

- Enganando o sistema (do inglês, Cheating the system): onde as regras do sistema não são claras, o que permite que os jogadores ignorem-as ou utilize brechas para obter vitória;

- Privacidade: onde o monitoramento e vigilância dos dados podem ocasionar brechas de seguranças quanto às informações do sistema, violando direitos de privacidade básicos do usuário;

- Efeitos declinantes: pode ocorrer quanto o efeito da gamificação se desgasta com o tempo, devido a falta de inovação ou complexidade dos desafios.

Apesar de haver um mapeamento inicial, estes problemas foram mapeados em contextos diferentes do educacional. Além disso, nem todos possuem evidências empíricas que os reforçam como problemas (Kim \& Werbach. 2016), nem podem ser considerados diretamente como parte do planejamento e/ou implementação.

\section{Materiais e métodos}

Para realizar esta pesquisa foi desenvolvida uma revisão sistemática, buscando estudos secundários (Revisões sistemáticas e de literatura) com foco na área de educação. Foram selecionados estudos secundários por conterem uma coletânea de trabalhos primários, e que passaram por um controle de qualidade durante a realização do estudo (no caso de estudos sistemáticos).

Para realizar a revisão foi utilizado o protocolo desenvolvido por Kitchenham (2004) que consiste em três etapas: Planejamento, onde são definidas as questões de 
pesquisa, strings de busca, critérios de inclusão e exclusão e bases de coleta; Condução, onde é realizada a busca nas bases selecionadas e aplicação dos critérios de inclusão e exclusão, a fim de selecionar os estudos a serem realizados; Relatório de resultados, onde é realizado o resumo dos resultados encontrados.

$\mathrm{Na}$ fase de planejamento foi definida a seguinte pergunta de pesquisa "Quais as principais dificuldades relacionadas ao planejamento e implantação da gamificação em contextos educacionais?". Com relação a busca, foram utilizados termos referentes a estudos secundários e gamificação como "Gamification", "Meta-analysis", "Systematic mapping", "Systematic Review" e "Literature review". Em seguida foram definidos os critérios de inclusão e exclusão (Tabela 1) para auxiliar na filtragem dos estudos selecionados.

Tabela 1: critérios de inclusão e exclusão

\begin{tabular}{|l|l|}
\hline Critérios de inclusão & Critérios de exclusão \\
\hline Ser do contexto educacional & Fora do contexto educacional \\
\hline Estar em inglês & Linguagem diferente do inglês \\
\hline $\begin{array}{l}\text { Estar publicado em conferência, capítulo } \\
\text { de livro ou periódico }\end{array}$ & Literatura cinza \\
\hline $\begin{array}{l}\text { Ser um estudo secundário (revisão da } \\
\text { literatura ou sistemática) }\end{array}$ & \\
\hline
\end{tabular}

Por fim, foram escolhidas bases de pesquisa de referência na área, sendo: IEEE Xplore, ACM Digital Library, Scopus, Engineering Village, Web of Science e ScienceDirect. Após a fase de Planejamento, foi iniciada a fase de Condução (Figura 1), onde a string foi utilizada nas bases de busca. A partir destes termos foram retornados 768 artigos, que foram analisados pelos seus títulos, resumos e palavras-chaves para identificar quais seriam utilizados para serem analisados. 


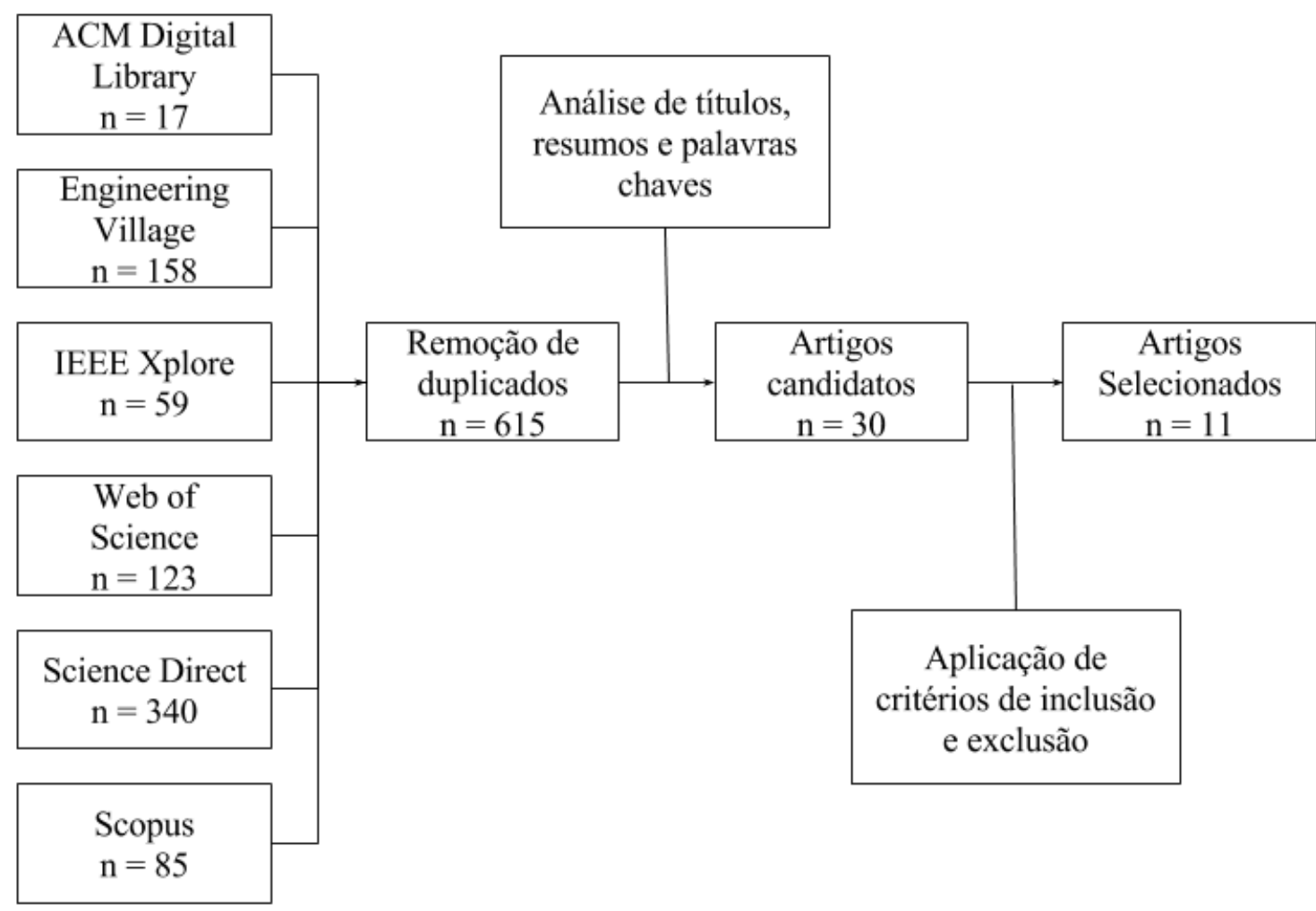

Figura 1: Fases da etapa de condução

Após esta análise, foram identificados 30 trabalhos candidatos que seriam lidos por completo. Em seguida, foram aplicados os critérios de inclusão e exclusão para identificar os estudos que seriam utilizados na pesquisa, totalizando 11 artigos (Borges et al., 2014; Dicheva et al., 2015; Faiella \& Ricciardi, 2015; Markopoulos et al., 2015; Azmi, Iahad \& Ahmad, 2015; Surendeleg et al., 2014; Al-Smadi, 2015; Busarello et al., 2016; Nah et al., 2014; Caponetto et al., 2014; Samungam et al., 2015). Estes artigos consistem de estudos secundários que abordam a gamificação em contextos educacionais, no entanto diferem quanto ao contexto que são explorados, como aprendizagem colaborativa.

\section{Resultados e discussões}

Nos onze artigos analisados, foram extraídas informações referentes aos estudos primários e os problemas reportados no planejamento e implantação da gamificação nos contextos de ensino. No total, foram identificados seis problemas relacionados à implantação da gamificação em contextos educacionais, sendo: Carência de evidências empíricas, Carências de métodos específicos, Customização, Comportamento indesejado, Carência de definições e Carência de suporte tecnológico.

Carência de evidências empíricas é caracterizada quando estudos primários analisados não fornecem evidências suficientes para atestar os efeitos benéficos da gamificação implantada. Este problema afeta a credibilidade da gamificação, uma vez que não há uma conexão entre prática e teoria (Seaborn \& Fels, 2014). Este problema foi reportado por sete estudos (Borges et al., 2014; Dicheva et al., 2015; Faiella \& Ricciardi, 2015; Markopoulos et al., 2015; Azmi, Iahad \& Ahmad, 2015; Surendeleg et al, 2014; Caponetto et al., 2015). Um ponto importante neste tópico é quando estudos V. $15 \mathrm{~N}^{\circ}$ 2, dezembro, 2017 
primários visam analisar e/ou mensurar comportamentos como motivação e engajamento, mas não apresentam o aporte teórico adequado para avaliarem isto.

Carência de métodos específicos foi identificado por quatro estudos secundários, é relacionado como a necessidade de métricas e ferramentas de avaliação bem definidas. Isto é uma preocupação recorrente em estudos primários, uma vez que a carência de métodos de avaliação prejudicam na avaliação da efetividade da gamificação que foi aplicada. Vários estudos primários focam em avaliar diversas métricas, motivação sendo a principal, porém utilizando instrumentos diferentes e não validados (Dicheva et al., 2015; Faiella \& Ricciardi, 2015; Azmi, Iahad \& Ahmad, 2015; Nah et al., 2014). Outro ponto levantado neste problema é a discrepância quanto aos instrumentos utilizados, quando há um instrumento validado utilizado, muitos dos instrumentos são feitos de forma ad-hoc sem a validação apropriada, ou não condizem com as teorias de motivação e engajamento que estão sendo utilizadas como base.

Customização é outro problema reportado em estudos secundários de gamificação, é relacionado à propriedade de modificar a experiência do usuário baseado no perfil (demográfico, de jogador ou outro) e/ou suas interações com o sistema gamificado (sistemas tutores inteligentes). Este problema foi reportado por três estudos (Dicheva et al., 2015; Faiella \& Ricciardi, 2015; Al-Smadi, 2015). Outro ponto importante relacionado a este problema é a extração e identificação destes perfis. A literatura de jogos apresenta alguns modelos de perfis de jogadores, no entanto nem todos podem ou devem ser utilizados como base para sistemas gamificados.

Comportamento indesejado é uma preocupação em estudos primários de gamificação, é relacionado às mudanças de comportamento que não foram previstas quando a gamificação foi planejada. Dentre estes comportamentos indesejados, os mais citados são uma diminuição na motivação, onde a gamificação provocou o efeito oposto do desejado, ou competição indesejada, onde o processo que foi gamificado gerou uma competição não saudável entre os usuários (Busarello et al., 2016; Sanmugan et al., 2015). Um ponto relevante relacionado a este problema é que algumas abordagens tentaram mapear o comportamento dos usuários das aplicações que intenciona-se gamificar, no entanto em contextos de ensino há uma dificuldade maior neste aspecto, devido a heterogeneidade dos perfis do público-alvo.

Carência de definições foi reportado por dois estudos, é relacionada a carência ou confusão entre os pesquisadores para definir ou expor conceitos básicos de gamificação. Isto foi considerado um problema, uma vez que a carência de termos atrapalha consideravelmente na implantação da gamificação, um exemplo disso são estudos que consideram pontos e conquistas como elementos (Hanus \& Fox, 2014; Kapp, 2012; Toda et al., 2014), em outros estudos estes são chamados de recompensas (Lee \& Hammer, 2011; Korn \& Schmidt, 2015; Markoupolos et al., 2015), ou diferenciam em outras classificações, como elementos de feedback (Kapp, 2012; Toda et al., 2016; Thiebes, Lins \& Basten, 2014). Foi reportado por dois estudos (Dicheva et al., 2015; Caponetto et al., 2015).

Essa carência de definições também influencia no desenvolvimento e elaboração de estratégias, uma vez que a discrepância entre os termos pode confundir profissionais do ensino, principalmente os que não tem costume de utilizá-los em seu dia-a-dia. A elaboração de estratégias, assim como o seu registro, é importante para o 
desenvolvimento de estudos na área de gamificação, uma vez que o mal mapeamento e definição dos termos também pode prejudicar os design de experimentação para obtenção de evidências empíricas.

Outra preocupação, destacada apenas por Dicheva et al (2015) é a carência de suporte tecnológico que pode atrapalhar ou até mesmo impedir o uso da gamificação em contextos educacionais. Isso é um fator debatido entre os estudos de gamificação, uma vez que há um consenso sobre a quantidade de informações que é possível extrair em ambientes gamificados (Heilbrunn, Herzig \& Schill, 2014).

Isso é uma preocupação importante, uma vez que vem surgindo interesse por parte dos profissionais do ensino em implantar a gamificação, no entanto não conseguem pela falta de tempo e recursos (Sánchez-Mena e Martí-Parreño, 2016; Martí-Parreño, Seguí-Mas e Seguí-Mas, 2016). É possível encontrar algumas ferramentas que apoiam etapas do processo de planejamento e implantação da gamificação (Heillbrun, Herzig \& Schill, 2014), no entanto nenhuma delas apoia o processo de design do início ao fim.

Com o intuito de realizar uma análise sobre os problemas mais frequentes (respectivamente, Carência de evidências empíricas [CarEvdEmp], Carência de métodos específicos [CarMetEsp], e Customização - [Custom]) foi gerado um diagrama de Venn (Herbele et al., 2015) apontando a intersecção entre os obstáculos relatados, na visão dos estudos secundários analisados (Figura 2).

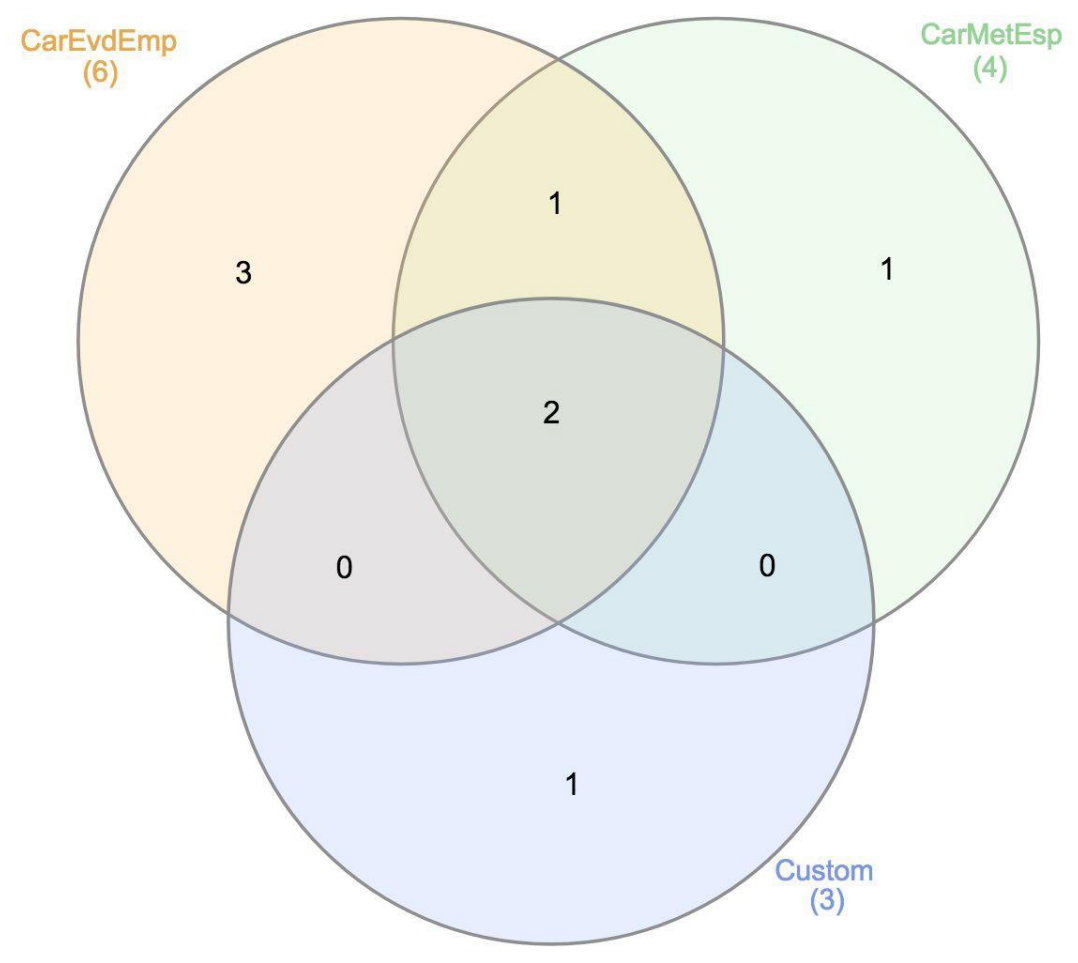

Figura 2: Diagrama de Venn representando a interseção entre os problemas relatados 
A partir deste gráfico (Figura 2), pode ser observado que a carência de métodos específicos tem um estudo relacionado com a carência de evidências empíricas, isso deve ocorrer pelo fato de não haver um consenso quanto a métodos e designs de experimentação nos estudos primários de gamificação, o que dificulta na avaliação.

Outra relação é quanto a customização, onde os autores reportam a necessidade de customizar os ambientes gamificados com os perfis de usuário. No entanto, a falta de instrumentos para a mensuração destes perfis e a forma de como avaliá-los também influencia negativamente no resultado empírico dos estudos.

A partir dos resultados encontrados, podemos direcionar pesquisas futuras para o desenvolvimento de modelos e instrumentos de avaliação para as abordagens de gamificação existentes. Uma possível proposta é o desenvolvimento de um modelo que, em conjunto com ferramentas inteligentes de autoria, possa auxiliar pesquisadores e profissionais de ensino na medição dos comportamentos esperados, como motivação e engajamento, e na extração dos perfis de seu público-alvo, para auxiliar na customização.

\section{Conclusões, Limitações e Trabalhos Futuros}

A gamificação é uma aliada poderosa para melhorar o engajamento e motivação de alunos, no entanto, para alcançar estes benefícios é necessário realizar um bom planejamento. Este trabalho apresentou uma lista de ameaças, com base em uma revisão da literatura aprofundada, que podem prejudicar a implantação da gamificação em contextos educacionais. Dentre os problemas encontrados, pode-se destacar a carência de evidências empíricas como o principal dos problemas, uma vez que foi reportado por sete estudos, seguido pela Carência de métodos específicos reportada por quatro estudos, isto significa que estudos primários devem ter uma atenção maior na preparação do design experimental, como as guidelines presentes em Klock et al (2015).

Dentre as limitações do trabalho pode-se citar o critério de inclusão de artigos somente no idioma inglês, isto é considerado uma limitação uma vez que exclui artigos nacionais publicados em periódicos e conferências indexados nas bases escolhidas. A não utilização de bases nacionais para a realização das buscas ocorreu pelo fato destas não aceitarem strings de busca como parte do seu mecanismo de busca, dificultando a coleta de artigos.

Este trabalho também apresenta uma contribuição para a área de gamificação, a partir do mapeamento dos problemas encontrados, uma vez que isto ainda não foi encontrado na literatura. Como trabalhos futuros, espera-se utilizar estes problemas para desenvolver critérios para avaliar os frameworks, métodos e processos de gamificação, com foco na educação, existentes. Além disso, outro foco de trabalho futuro é realizar uma inclusão manual de estudos secundários encontrados em bases nacionais e outros que não aceitam recursos de busca com strings lógicas, como o Google Scholar. 


\section{Agradecimentos}

Os autores gostariam de agradecer ao CNPq, Capes e a Fundação de Amparo à Pesquisa do Estado de São Paulo (FAPESP Processo 2016/02765-2) pelo apoio financeiro.

\section{Referências}

ANDRADE, F. R.H; MIZOGUCHI, R.; ISOTANI, S. The bright and dark sides of gamification. Proceedings of the International Conference on Intelligent Tutoring Systems, p. 176-186, 2016.

BORGES, S. DE S. et al. A systematic mapping on gamification applied to education. Proceedings of the 29th ACM Symposium on Applied Computing, p. 216-222, 2014.

DETERDING, S. et al. From Game Design Elements to Gamefulness: Defining "Gamification". Proceedings of the ACM annual conference on Human factors in Computing Systems - CHI, p. 9-15, 2011.

DICHEVA, D. et al. Gamification in Education : A Systematic Mapping Study. Educational Technology \& Society, v. 18, n. 3, p. 75-88, 2015.

ZICHERMANN, G.; CUNNINGHAM, C. Gamification by Design: Implementing Game Mechanics in Web and Mobile Apps. [s.1.] O'Reilly Media; 1 edition, 2011.

KAPP, K. M. The Gamification of Learning and Instruction: Game-based Methods and Strategies for Training and Education, 2012.

TODA, A. M. et al. Desenvolvimento de uma aplicação web para auxiliar no ensino da Matemática para alunos do Ensino Fundamental. XXV Simpósio Brasileiro de Informática na Educação (SBIE 2014), p. 392-401, 2014

KLOCK, A. C. T. et al. Análise das técnicas de Gamificação em Ambientes Virtuais de Aprendizagem. RENOTE, v. 12, n. 2, 2015.

TODA, A. et al. Um processo de Gamificação para o ensino superior: Experiências em um módulo de Bioquímica. Anais do Workshop de Informática na Escola, p. 495-504, 2016.

CHALLCO, G. C.; MIZOGUCHI, R.; ISOTANI, S. An Ontology Framework to Apply Gamification in CSCL Scenarios as Persuasive Technology. Revista Brasileira de Informática na Educação, v. 24, n. 2, p. 67, 2016.

SEABORN, K.; FELS, D. I. Gamification in Theory and Action: A Survey. Internatoinal Journal of Human-Computer Studies, v. 74, p. 14-31, 2014.

HANUS, M. D.; FOX, J. Assessing the effects of gamification in the classroom: A longitudinal study on intrinsic motivation, social comparison, satisfaction, effort, and academic performance. Computers \& Education, v. 80, p. 152-161, 2014.

LEE, J. J.; HAMMER, J. Gamification in Education: What, How, Why Bother? Academic Exchange Quarterly, v. 15, p. 1-5, 2011.

KORN, O.; SCHMIDT, A. Gamification of Business Processes: Re-designing Work in Production and Service Industry. Procedia Manufacturing, v. 3, p. 3424-3431, 2015.

MARKOPOULOS, A. P. et al. Gamification in engineering education and professional training. International Journal of Mechanical Engineering Education, v. 43, n. 2, p. 118-131, 2015.

THIEBES, S.; LINS, S.; BASTEN, D. Gamifying information systems A synthesis of gamification mechanics and dynamics. Proceedings of the 22nd European Conference on Information Systems, p. 1-17, 2014. 
HEILBRUNN, B.; HERZIG, P.; SCHILL, A. Tools for Gamification Analytics: A Survey. Proceedings of the IEEE/ACM 7th International Conference on Utility and Cloud Computing, p. 603-608, 2014

FAIELLA, F.; RICCIARDI, M. Gamification and learning: A review of issues and research. Journal of E-Learning and Knowledge Society, v. 11, n. 3, p. 13-21, 2015.

AZMI, S.; IAHAD, N. A.; AHMAD, N. Gamification in online collaborative learning for programming courses: A literature review. v. 10, n. 23, p. 18087-18094, 2015.

SURENDELEG, G. et al. The role of gamification in education-a literature review. Contemporary Engineering Sciences, v. 7, n. 29-32, p. 1609-1616, 2014.

AL-SMADI, M. GAMEDUCATION: Using Gamification Techniques to Engage Learners in Online Learning. Communications in Computer and Information Science, v. 486, p. 85-97, 2015.

BUSARELLO, R. I. et al. Gamification Approaches to Learning and Knowledge Development: A Theorical Review. In: Advances in Intelligent Systems and Computing. [s.1.] Springer International Publishing, 2016. v. 444p. 1107-1116.

NAH, F. F.-H. H. et al. Gamification of education: A review of literature. Lecture Notes in Computer Science (including subseries Lecture Notes in Artificial Intelligence and Lecture Notes in Bioinformatics). Anais...Springer International Publishing, p. 401-409 2014.

CAPONETTO, I.; EARP, J.; OTT, M. Gamification and Education: A Literature Review. Proceedings of the European Conference on Games Based Learning, v. 1, n. 2009, p. 50-57, 2014.

SANMUGAM, M. et al. Gamification as an educational technology tool in engaging and motivating students; An analyses review. Advanced Science Letters, v. 21, n. 10, p. 3337-3341, 2015.

NACKE, L. E.; DETERDING, S. The maturing of gamification research. Computers in Human Behavior, v. 71, p. 450-454. 2017.

KIM, T. W.; WERBACH, K. More than just a game: ethical issues in gamification. Ethics and Information Technology, v. 18, n. 2, p. 157-173. 2016.

HEBERLE, H. et al. InteractiVenn: a web-based tool for the analysis of sets through Venn diagrams. BMC Bioinformatics, v. 16, n. 1, p. 169. 2015.

SÁNCHEZ-MENA, A.; MARTÍ-PARREÑO, J. Gamification in higher education: teachers' drivers and barriers. Proceedings of the International Conference of The Future of Education, p. 180-184, 2016.

MARTÍ-PARREÑO, J.; SEGUÍ-MAS, D.; SEGUÍ-MAS, E. Teachers' Attitude towards and Actual Use of Gamification. Procedia - Social and Behavioral Sciences, v. 228, p. 682-688, 2016.

KIM, T. W.; WERBACH, K. More than just a game: ethical issues in gamification. Ethics and Information Technology, v. 18, n. 2, p. 157-173. 2016. 